\title{
ESTUDO COM GESTANTES ATENDIDAS EM UM CENTRO MUNICIPAL DE SAÚDE DA PERIFERIA DE BOTUCATU - SÃO PAULO - RELAÇÃO ENTRE BAIXO PESO MATERNO E BAIXO PESO AO NASCER DO CONCEPTO
}

\author{
Cristina Maria Garcia de Lima * \\ Nilza Teresa Rotter Pelá **
}

\begin{abstract}
Este trabalho teve o objetivo de estudar um grupo de 15 gestantes de baixo peso, que fizeram pré-natal em um Centro Municipal de Saúde da periferia de Botucatu - SP, triadas pela "Curva de Rosso", comparando-as a um grupo de 08 gestantes de peso normal triadas pelo mesmo instrumento e que freqüentavam o mesmo serviço pré-natal. Verificouse que os filhos de mulheres de peso normal pesaram, em média, $118 \mathrm{~g}$ a mais que os filhos das mulheres de baixo peso; independente do peso materno, pesaram mais os recémnascidos de mulheres com altura superior a $155 \mathrm{~cm}$ e não fumantes; as mulheres de peso normal tiveram, em média, 2 semanas de gestação a mais; entre os filhos das gestantes de baixo peso observamos relação entre peso médio ao nascer e ascensão na curva altura uterina $x$ idade gestacional; não houve relação entre anemia materna e intervalo interpartal inferior a 2 anos e peso ao nascer.
\end{abstract}

UNITERMOS: nutrição, gestantes, baixo peso

\section{INTRODUÇÃO}

A gestação acarreta unia modificação nutricional para a futura mãe, não só para corresponder às suas necessidades nutricionais, como também às do feto (MITCHEL et al., 1978).

\footnotetext{
*Professor Assistente do Curso de Enfermagem da Faculdade de Medicina de Botucatu área de Enfermagem Ginecológica, Obstétrica e Neonatal

**Professor Titular da Escola de Enfermagem de Ribeirão Preto da Universidade de São Paulo
} 
Vários autores têm apontado a existência de uma relação entre o estado nutricional da gestante e o peso ao nascer do recém-nascido. Assim, SINISTERR.A RODRIGUEZ et al. (1991) em estudo desenvolvido em São Paulo encontraram a incidência de 23,6\% de baixo peso ao nascer (menor ou igual a $2.500 \mathrm{~g}$ ) entre filhos de parturientes desnutridas, valor que caiu a 10,8\% no grupo de mulheres não desnutridas no final da gestação. MITCHEL, et al. (1978) apresentam o trabalho desenvolvido por Burke e colaboradores nos Estados Unidos, onde 95\%das crianças nascidas de mães que recebiam dietas excelentes ou boas, tiveram peso excelente ou bom ao nascimento; este número diminuiu a $53 \%$ quando as mães tinham dietas regulares e $8 \%$ quando suas dietas eram deficientes ou muito deficientes. SIQUEIRA et al. (1975) apontam que a desnutrição materna prejudica o crescimento do feto, tanto em peso como altura, aumentando o risco de morbi-mortalidade perinatal.

O crescimento fetal deficiente geralmente manifesta-se pelo baixo peso ao nascer e gera conseqüências imediatas, como o aumento da morbi-mortalidade perinatal e tardio, como as deficiências nutricionais, os processos patológicos e os distúrbios do crescimento (SIQUEIRA et al., 1985). Estudo realizado na Guatemala, com avaliação Iongitudinal mostrou que aos 6 meses de vida as crianças têm uma relação inversa entre desenvolvimento motor e baixo peso ao nascer, embora tal discrepância tenha diminuído com o passar dos anos (PEREZ-ESCAMILLA, POLLITT, 1992). SIQUEIRA et al. (1975 b) encontraram um risco de morte antes de 28 dias de vida 14 vezes maior entre recémnascidos de baixo peso, quando comparados com os demais.

A importância do baixo peso ao nascer é também quantitativa. Em 1980 a Organização Mundial de Saúde - OMS, estimou que 17\% dos nascidos em todo mundo apresentavam baixo peso, sendo que $94 \%$ destes nascimentos ocorriam em países em desenvolvimento (PEREZ-ESCAMILLA, POLLITT, 1992).

Para a SECRETARIA DE SAÚDE DO ESTADO DE SÃO PAULO (1990), o baixo peso ao nascer em populações desfavorecidas do ponto de vista sócio-econômico relaciona-se com a desnutrição materna e o ganho de peso insuficiente durante a gestação. Desta forma, avaliação do estado nutricional e ganho de peso da gestante é indispensável para que se detecte situações de risco, visando uma intervenção eficaz. O Ministério da Saúde em seus manuais sobre assistência pré-natal (BRASIL, MS, 1986, BRASIL, M.S., 1988) propõe, para a avaliação do estado nutricional da gestante, a utilização da "Curva de Rosso", desenvolvida a partir de estudos realizados com gestantes americanas e chilenas em 1985 e já validada para uso no Brasil (S. Paulo, S.E.S., 1990).

A Secretaria de Saúde do Estado de São Paulo também indica a utilização desse instrumento para avaliação do estado nutricional e ganho de peso da gestante (SÃO PAULO, S.E.S., 1990).

Este trabalho visa estudar um grupo de gestantes de baixo peso, triadas pelo instrumento "Curva de Rosso", buscando uma relação com o peso ao nascer de seu filho e também com patologias intercorrentes à gravidez, curva altura uterina x idade gestacional, 
intervalo interpartal, tabagismo e idade gestacional no momento do parto. Tal estudo tem por finalidade fornecer subsídios para, conhecendo a realidade das gestantes atendidas, elaborar uma "Proposta de Atendimento Nutricional às Gestantes" do Centro Municipal de Saúde no qual o estudo foi realizado.

\section{MATERIAL E MÉTODO}

\subsection{O local de realização do estudo}

O Centro Municipal de Saúde do Jardim Peabirú localiza-se na periferia do município de Botucatu - SP e atende uma população predominantemente de trabalhadores rurais e migrantes, com baixa renda familiar, sem residência própria, fazendo com que permaneçam pouco tempo na mesma residência ou região ${ }^{\star \star \star}$.

\subsection{População do estudo}

O estudo foi realizado com mulheres que fizeram pré-natal no Centro Municipal de Saúde do Jardim Peabirú e que já haviam dado a luz no momento em que iniciamos este trabalho.

Ressaltamos que o atendimento pré-natal nesta Unidade de Saúde é desenvolvido através de consultas de enfermagem (realizadas pela autora desde setembro/92), intercaladas com pelo menos duas consultas médicas: uma no início do acompanhamento pré-natal e outra no final desse, conforme preconizado pelo Ministério da Saúde (BRASIL, M. S., 1986).

O grupo estudado foi composto por 15 gestantes, classificadas no primeiro atendimento como de baixo peso e que permaneceram nesta classificação até o momento do parto. Não foram incluídas as mulheres que iniciaram o processo gravídico na categoria A (baixo peso), mas que se recuperaram durante o pré-natal, pois trabalhos como ode SHMISTERRA - RODRIGUEZ et al. (1991) apontam que a incidência de recém-nascidos de baixo peso é significativamente maior (2,8 vezes) entre as gestantes que não se recuperam durante a gravidez, quando comparadas àquelas que se recuperam.

O grupo controle foi composto por 8 mulheres que na primeira consulta foram categorizadas como B (peso normal), tendo permanecido nesta classificação até o momento do parto.

${ }^{\star * \star}$ FONTE: GERALDO, O.S. et al. Estudo da morbilidade na demanda do Centro Municipal de Saúde do Jardim Peabirú. Botucatu: Faculdade de Medicina de Botucatu - UNESP, 1989 (mimeografado) 


\subsection{A coleta de dados}

Para classificação da gestante quanto a seu estado nutricional, utilizou-se a "Curva de Rosso", a qual usa como indicador o percentual peso/altura, em relação ao padrão de referência, nas diversas idades gestacionais, dispensando o conhecimento do peso anterior à gestação. O padrão de referência, por sua vez, expressa o percentual de peso ideal para altura e idade gestacional, ajustado para compleição física média (SÃO PAULO, S.E.S., 1990).

Desta forma, a cada consulta, utiliza-se um "Nomograma" que permite a classificação da relação peso/altura da mulher em porcentagem; a seguir, anota-se o valor obtido em um gráfico de aumento de peso para gestantes, permitindo-se a categorização do estado nutricional da grávida em: $A=$ baixo peso; $B$ peso normal; $C=$ sobre peso; $D=$ obesidade (SÃO PAULO, S.E.S., 1990). O nomograma e gráfico utilizados para registro dos dados bem como a curva para acompanhamento do crescimento fetal, são apresentados no Anexo 1.

Os pontos obtidos a cada atendimento são ligados, conseguindo-se o traçado do peso da gestante, cuja inclinação dá a orientação quanto à adequação ou não do ganho de peso durante a gravidez. As condutas preconizadas pelo Ministério da Saúde (BRASIL. M. S., 1988) e Secretaria de Saúde do Estado de São Paulo (SÃO PAULO, S.E.S., 1990) a serem adotados já a partir da primeira consulta, após afasta-se a possibilidade de erro da idade gestacional, depende de uma avaliação pontual, no momento do primeiro atendimento e do traçado do peso da gestante obtido com os retornos subseqüentes (Anexo 2).

O instrumento utilizado para coleta dos dados analisados neste estudo é apresentado no Anexo 3.

\section{RESULTADOS E DISCUSSÃO}

Os resultados obtidos são apresentados a seguir. Buscaremos relacionar o peso ao nascer do recém-nascido como: estatura e estado nutricional da mãe, intervalo interpartal e curva da altura uterina $x$ idade gestacional. Estudaremos também a relação entre o estado nutricional da gestante e a idade gestacional da criança ao nascer.

\subsection{Estado nutricional/peso ao nascer}

A relação entre o estado nutricional da gestante e o peso ao nascer do recémnascido vem sendo descrita por autores como PEREZ-ESCAMILLA; POLLITT. (1992), LECHTIG et al. (1977), SIQUEIRA et al. (1985), SINISTERRA-RODRIGUEZ et al. (1991), 
BENZECRY, ACCIOLY (1989), SIQUEIRA et al. (1975 c) entre outros.

Em nosso trabalho, a diferença de peso média entre os filhos de mulheres de baixo peso e os de mulheres com peso normal foi de $118 \mathrm{~g}$ a mais para os filhos daquelas com peso normal.

Entretanto, entendemos que é difícil precisar exatamente o papel que o baixo peso materno desempenha sobre o peso ao nascer e morbi-mortalidade infantil, já que geralmente associam-se desnutrição materna crônica, outras patologias maternas e outros problemas relacionados com a pobreza.

\subsection{Estatura materna/peso ao nascer}

O Quadro 1 apresenta a relação estatura materna/peso ao nascer do recém-nascido.

QUADRO 1 - RELAÇÃO ENTRE ESTADO NUTRICIONAL DA GESTANTE, ALTURA MATERNA E PESO MÉDIO AO RECÉM-NASCIDO AO NASCER

\begin{tabular}{|c|c|c|}
\hline $\begin{array}{c}\text { Estado Nutricional } \\
\text { da Gestante }\end{array}$ & $\begin{array}{c}\text { Altura Materna } \\
(\mathrm{cm})\end{array}$ & $\begin{array}{c}\text { Peso Médio do } \\
\text { Recém-Nascido }(\mathrm{g})\end{array}$ \\
\hline Baixo Peso & $<=155$ & 2.860 \\
\cline { 2 - 3 } & $>155$ & 3.255 \\
\hline Peso Normal & $<=155$ & 2.893 \\
\cline { 2 - 3 } & $>155$ & 3.308 \\
\hline
\end{tabular}

Verificamos que as mulheres com altura superior a $155 \mathrm{~cm}$ tiveram filhos com maior peso, independente de seu estado nutricional (395g a mais entre as gestantes com baixo peso e $415 \mathrm{~g}$ entre as de peso normal). A Figura 1 mostra estas relações. 
FIGURA 1 - PESO AO NASCER DO RECÉM-NASCIDO SEGUNDO A ALTURA E ESTADO NUTRICIONAL DA MÃE

Figura 1

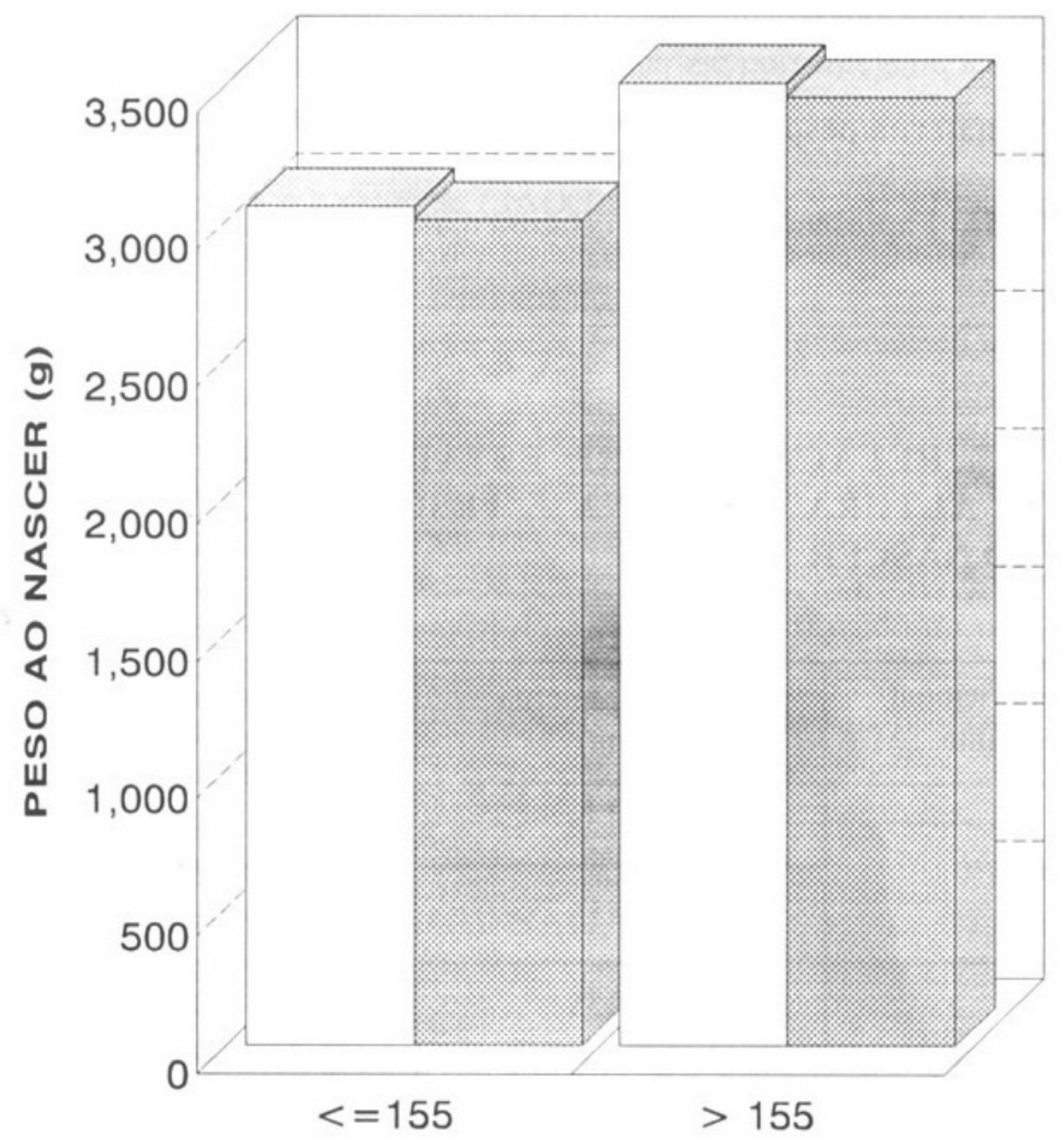

ALTURA MATERNA (CM)

\section{GEST.DE BAIXO PESO GEST. DE PESO NORMAL}


Assim, os dados por nós obtidos são concordantes com a literatura, já que autores como STEFANINI et al. (1988), CIARI JR. et al. (1975). SIQUEIRA et al. (1975 b) e LECHTIG et al. (1977) observaram que a altura materna associa-se ao peso natal, já que mães menores tendem a ter filhos menores.

\subsection{Anemia materna/peso ao nascer}

$\mathrm{Na}$ literatura, estudos sobre o baixo peso ao nascer têm procurado verificar se este relaciona-se com a anemia materna. Em nosso trabalho, entre as gestantes com baixo peso e anêmicas ( $\mathrm{Hb}$ menor que 11,0 g/dl), o peso médio de seus recém-nascidos ao nascer foi $3.025 \mathrm{~g}$ e, portanto, apenas $9 \mathrm{~g}$ abaixo da média encontrada para o grupo de recém-nascidos de mães com baixo peso. Entre as gestantes com peso normal e anêmicas, o peso médio de seus filhos, foi de $3.240 \mathrm{~g}$ e, portanto, $88 \mathrm{~g}$ acima da média esperada.

Assim, em nossa amostra, não pudemos observar relação entre anemia materna e baixo peso.

Para SINISTERRA-RODRIGUEZ (1989), entre os fatores que interferem na qualidade da gravidez e, conseqüentemente, no peso ao nascer, destacam-se as deficiências nutricionais, entre elas a anemia, não só por sua prevalência elevada, mas também pelos efeitos adversos a ela associados. Entretanto, em trabalho realizado em maternidade assistencial de São Paulo, não observaram associação entre anemia e baixo peso ao nascer, mesmo quando utilizaram para identificação de anemia 9,5g Hb/dl (anemia severa) ou peso ao nascer de $3.000 \mathrm{~g}$ (peso ao nascer inadequado).

Ressaltamos que a anemia foi a única patologia intercorrente a gravidez observada nos dois grupos de mulheres estudadas.

\subsection{Intervalo interpartal/peso ao nascer}

Não observamos, entre as gestantes estudadas, relação entre intervalo interpartal inferior a 2 anos e baixo peso ao nascer do recém-nascido, independentemente do estado nutricional materno. Em ambos os grupos: gestantes com baixo peso e gestantes normais, o peso médio dos recém-nascidos cujas mães tinham história de parto há menos de 2 anos foi superior ao peso médio encontrado para cada um dos grupos. 
Entretanto, para BENZECRY, ACCIOLY (1989) gestações sucessivas em curto espaço de tempo implicam em um esgotamento do organismo materno, sob o ponto de vista nutricional, aumentando as possibilidades de crianças com baixo peso e conseqüente aumento da mortalidade infantil.

\subsection{Tabagismo/peso ao nascer}

Para gestantes fumantes, independentemente do seu estado nutricional, pudemos observar um peso médio do recém-nascido inferior ao encontrado para o grupo como um todo. Desta forma, os recém-nascidos de mulheres fumantes e de baixo peso, pesaram em média $97 \mathrm{~g}$ a menos, ao passo que aqueles nascidos de mulheres fumantes e com peso normal, pesaram $309 \mathrm{~g}$ a menos, quando comparados a mulheres não fumantes.

Estudos como o de SIQUEIRA et al. (1985) e PEREZ-ESCAMILLA, POLLITT (1992) também apresentam esta relação.

\subsection{Estado nutricional/idade gestacional do recém-nascido ao nascer}

A idade gestacional média observada para gestantes de baixo peso foi de 38 semanas e um dia, ao passo que para as gestantes com peso normal, a duração média da gravidez foi de 40 semanas e um dia. Assim, as gestantes com peso normal tiveram em média 2 semanas de gestação a mais, quando comparadas com aquelas de baixo peso. Por outro lado, a idade gestacional vem sendo apontada como um dos principais fatores determinantes do peso ao nascer por autores como LECHTIG et aí. (1977), SIQUEIRA et al. (1975 b) e CIARI JR. et al. (1975).

\subsection{Curva da altura uterina $\mathrm{x}$ idade gestacional/peso ao nascer}

Os dados desta avaliação foram obtidos do último atendimento da gestante antes do parto.

Para o grupo de gestantes com baixo peso, pudemos observar um aumento no peso médio do recém-nascido ao nascer, à medida que houve uma ascensão na curva da altura uterina $x$ idade gestacional, conforme apresentamos na figura 2. 
FIGURA 2 - PESO DO RECÉM-NASCIDO AO NASCER, SEGUNDO A CURVA DA ALTURA UTERINA X IDADE GESTACIONAL DAS GESTANTES DE BAIXO PESO

\section{Figura 2}

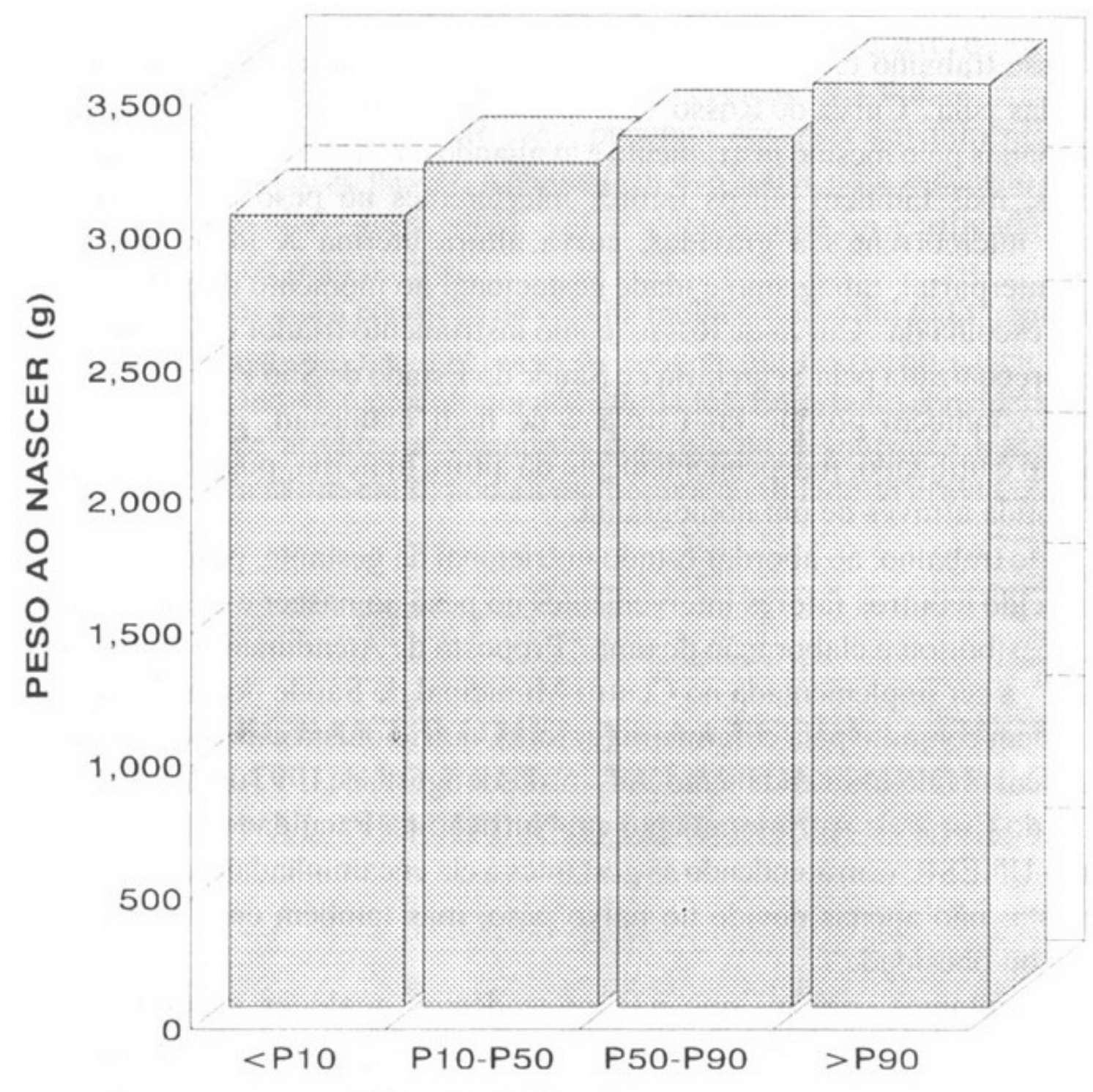

CURVA ALT.UTERINA x IDADE GEST. 
Ao observarmos a figura 2, podemos verificar que o gráfico utilizado para acompanhamento do crescimento intra-útero entre gestantes de baixo peso permitiu a identificação, ainda no período gestacional, de crianças com risco para o baixo peso ao nascer, Entretanto, tal associação não foi verificada entre gestantes com peso normal. Isso nos faz refletir sobre alguns pontos, como: maior facilidade de verificação da altura uterina em mulheres magras e necessidade de ampliarmos nossa amostra, a fim de que possamos comprovar a adequação da curva proposta a nossa realidade.

\section{CONSIDERAÇÕES FINAIS}

Esse trabalho teve o objetivo de estudar um grupo de gestantes de baixo peso, triadas pela "Curva de Rosso", comparando-as com um grupo de gestantes normais triadas pelo mesmo instrumento e avaliando não apenas o peso ao nascer de seus filhos, mas também outros fatores interferentes no peso ao nascer como: patologias intercorrentes a gravidez, curva altura uterina $x$ idade gestacional, intervalo interpartal, tabagismo e idade gestacional no momento do parto.

A escolha da "Curva de Rosso" como instrumento triador deveu-se ao fato desta ser preconizada pela Secretaria de Saúde do Estado de São Paulo e Ministério da Saúde e também porque tal Curva é de fácil manuseio, já que um único instrumento é utilizado, independentemente da altura materna, pois a relação peso/altura é obtida através de um nomograma.

Este trabalho, ao abordar estado nutricional da gestante, peso ao nascer do recémnascido e outros fatores intervenientes no peso ao nascer e já anteriormente citados, possibilitou a elaboração de uma "Proposta de Atendimento Nutricional a Gestantes", a ser implementada no Centro Municipal de Saúde do Jardim Peabirú e realizada em conjunto pela enfermeira (autora), nutricionista e médico sanitarista-nutrólogo, envolvidos no atendimento. Assim, desde agosto de 1993 uma nutricionista, estagiária do Curso de Aprimoramento em Nutrição da Faculdade de Medicina de Botucatu - UNESP, vem atendendo as gestantes a ela encaminhadas pela enfermeira pré-natalista, não apenas devido ao baixo peso, mas também em decorrência de sobrepeso ou obesidade.

Ressaltamos, porém, que não pretendemos qualquer generalização de nossos resultados, visto que por termos incluído tanto no grupo de estudo quanto no grupo controle apenas as mulheres que iniciaram e terminaram o processo gravídico na mesma classificação (baixo peso ou peso normal), o número amostrado foi relativamente pequeno. Pretendemos, assim, dar continuidade a este trabalho em um futuro próximo, para que possamos incluir um maior número de mulheres.

Resumindo os resultados obtidos, verificamos que: 
- a diferença de peso média entre os filhos de mulheres de baixo peso e os de mulheres com peso normal foi de $118 \mathrm{~g}$ a mais para os filhos daqueles com peso normal;

- os bebês das mulheres com altura superior a $155 \mathrm{~cm}$ pesaram mais que aqueles nascidos de mulheres mais baixas. A diferença foi de $395 \mathrm{~g}$ no grupo de baixo peso e $415 \mathrm{~g}$ no grupo de peso normal;

- a única patologia intercorrente à gravidez observada foi a anemia materna $(\mathrm{Hb}$, menor que 11,0g/dl). Entretanto, não houve relação entre anemia materna e baixo peso;

- não observamos relação entre intervalo interpartal inferior a 2 anos e baixo peso ao nascer, independentemente do estado nutricional materno;

- entre as gestantes fumantes, pudemos observar um peso médio ao nascer inferior ao encontrado para o grupo como um todo: $97 \mathrm{~g}$ a menos para os filhos de fumantes de baixo peso e $309 \mathrm{~g}$ a menos para os filhos de fumantes de peso normal;

- a idade gestacional média observada para gestantes de baixo peso foi de 38 semanas e 1 dia e para gestantes de peso normal foi de 40 semanas e 1 dia;

- para o grupo de gestantes de baixo peso, pudemos observar um aumento no peso médio do recém-nascido ao nascer, à medida que houve uma ascensão na curva da altura uterina $x$ idade gestacional. Tal associação não foi verificada entre os filhos de mulheres de peso normal.

Entendemos que a condição devida da mulher, como um todo, representa o grande fator interveniente nas condições de nascimento do recém-nascido, muito mais que os fatores individualmente por nós estudados. Entretanto consideramos que a implementação de atividades visando o controle da desnutrição materna, nos serviços de assistência prénatal, poderá melhorar a qualidade da gravidez e, assim, do recém-nascido.

\section{STUDY OF PREGNANT WOMEN AT A CITY HEALTH CENTER IN BOTUCATU, SÃO PAULO: RELATIONSHIP OF LOW MATERNAL WEIGHT AND LOW BIRTH WEIGHT}

This study evaluated a group of 15 low-weight pregnant women, who underwent prenatal medical examinations at a City Health Center on the outskirts of Botucatu - SP, using the "Rosso Curve ", and compared them with a group of 8 normal-weight pregnant women, also screened by the same institution at the same prenatal service. We verified that the children born from normal-weight pregnant women weighed an average $118 \mathrm{~g}$ more than those of low-weight pregnant women. Independent of the mother's weight, those born from mothers whose height was greater than $155 \mathrm{~cm}$ and were non-smokers weighed more. Pregnant women of normal weight had, on average, an additional 2 weeks of pregnancy: 
Amongst those born of low-weight pregnant women, we noted a connection between medium birth weight and an increase on the uterine height curve $x$ gestational age. There was no connection between maternal anemia and interpartal intervals less than to 2 years and birth weight.

UNITERMS: nutrition, pregnant women, low weight

\section{ESTUDIO CON GESTANTES ATENDIDAS EN UN CENTRO MUNICIPAL DE SALUD DE LOS ALREDEDORES DE BOTUCATU - SÃO PAULO - RELACIÓN ENTRE BAJO PESO MATERNO Y BAJO PESO AL NACER DEL CONCEPTO}

Este trabajo tuvo como objetivo estudiar un grupo de 15 gestantes de bajo peso que realizaron el prenatal en un Centro Municipal de Salud Pública de la periferia de Botucatu $S P$, seleccionadas a través de la "Curva de Rosso" a las cuales se comparó con un grupo de 8 gestantes de peso normal seleccionadas a través del mismo instrumento y que frecuentaban el mismo servicio prenatal. Con este estudio se verificó que los hijos de mujeres de peso normal tuvieron un peso promedio de $118 \mathrm{~g}$ más que los hijos de las mujeres de bajo peso; independiente del peso materno, pesaron más los recién nacidos de mujeres con talla superior a $155 \mathrm{~cm}$ y no fumadoras; las mujeres de peso normal tuvieron en promedio 2 semanas más de gestación que las mujeres de bajo peso. Entre los hijos de las gestantes de bajo peso observaron una relación entre el peso promedio al nacer y el aumento en la curva altura uterino por edad gestacional; no encontrándose ninguna relación entre anemia materna y los periodos ínter genésicos inferiores a 2 años el peso al nacer.

UNITERMOS: nutrición, gestantes, bajo peso

\section{REFERÊNCIAS BIBLIOGRÁFICAS}

01. BENZECRY, E.H.; ACCIOLLY, E. Nutrição materno-infantil. Rio de Janeiro: Cultura Médica, 1989. p. 69.

02. BRASIL. Ministério da Saúde. Secretaria Nacional de Programas Especiais de Saúde. Divisão Nacional de Saúde Materno-Infantil. Programa de Assistência Integral à Saúde da Mulher. Instituto de Assistência Médica da Previdência Social. Pré-natal de baixo risco. Brasília: Ministério da Saúde, 1986. p. 44. (Série A: normas e manuais técnicos, 36). 
03. BRASIL. Ministério da Saúde. Secretaria Nacional de Programas Especiais de Saúde. Divisão Nacional de Saúde Materno-Infantil. Programa de Assistência Integral à Saúde da Mulher. Instituto Nacional de Assistência Médica da Previdência Social. Assistência pré-natal. 2 ed. Brasília: Ministério da Saúde, 1988. p. 44. (Série A: normas e manuais técnicos, 36).

04. CIARI JR., C. et al. Relação entre peso da criança ao nascer, altura materna, idade gestacional e restrição alimentar em gestantes normais. Rev.Saúde Pública, São Paulo, v. 9, n. 1, p. 3342, 1975.

05. LECHTIG, A. et al. Os efeitos de uma nutrição aperfeiçoada, a partir da concepção da criança até os 3 anos de idade, na mortalidade infantil. J.Pediatr., Rio de Janeiro, v. 42, n. 1, p. 40-7, 1977.

06. MITCHELL, H.S. et al. Nutrição. 16 ed. Rio de Janeiro: Interamericana, 1978. p. 567.

07. PEREZ-ESCAMILLA, R.; POLLITT, E. Causas y consecuencias del retraso del crecimiento intrauterino en América Latina. Bol. Of. Sanit. Panam., v. 112, n. 6, p. 473-93, 1992.

08. SÃO PAULO. Secretaria de Estado da Saúde. Centro de Apoio ao Desenvolvimento da Assistência Integral à Saúde. Área Técnica de Atenção à Saúde da Mulher. Programa de Nutrição. Avaliação do estado nutricional da gestante e do ganho de peso durante a gestação. São Paulo: SES, 1990. p. 15.

09. SINISTERRA-RODRIGUEZ, O.T. et al. Anemia e desnutrição maternas e sua relação com o peso ao nascer. Rev. Saúde Pública, São Paulo, v. 25, n. 3, p. 193-7, 1991.

10. SINISTERRA-RODRIGUEZ, O.T. Estudo da influência da anemia e desnutrição maternas sobre peso ao nascer. São Paulo, p.75. Dissertação (Mestrado) Faculdade de Saúde Pública, Universidade de São Paulo, 1989.

11. SIQUEIRA, A.A.F. et al. Utilização de uma curva de decrescimento intra-uterino corrigido para peso e altura maternas. Rev. Saúde Pública, São Paulo, v. 9, n. 2, p. 215-20, 1975 a.

12. Influência da altura e ganho de peso materno e da idade gestacional sobre o peso do R. N.: estudo de 03 grupos de gestantes normais. Rev. Saúde Pública, São Paulo, v. 9, n. 3, p. 331-42, 1975 b.

13. A utilização de uma curva ponderal de gestantes normais no diagnóstico de desnutrição intra-uterina. Rev. Saúde Pública, São Paulo, v. 9, n. 4, p. 495-506, 1975 c.

14. Estado nutricional e hábito de fumar maternos, crescimento intra-uterino e pós-natal. Rev. Saúde Pública, São Paulo, v. 19, n. 1, p. 37-50, 1985.

15. STEFANINI, M.L.R. et al. Avaliação do programa de nutrição em saúde, no grupo de gestantes de sete municípios de São Paulo, Brasil. Rev. Nutr. PUCCAMP., Campinas, v. 1, n. 2, p. 153-62, jul/dez, 1988. 


\section{Anexo 1}

NOMOGRAMA PARA CLASSIFICAÇÃO DE RELAÇÃO PESO/ALTURA DE MUIIIER (\%)

GRÁFICO DE AUMENTO DE PESO PARA GESTANTES

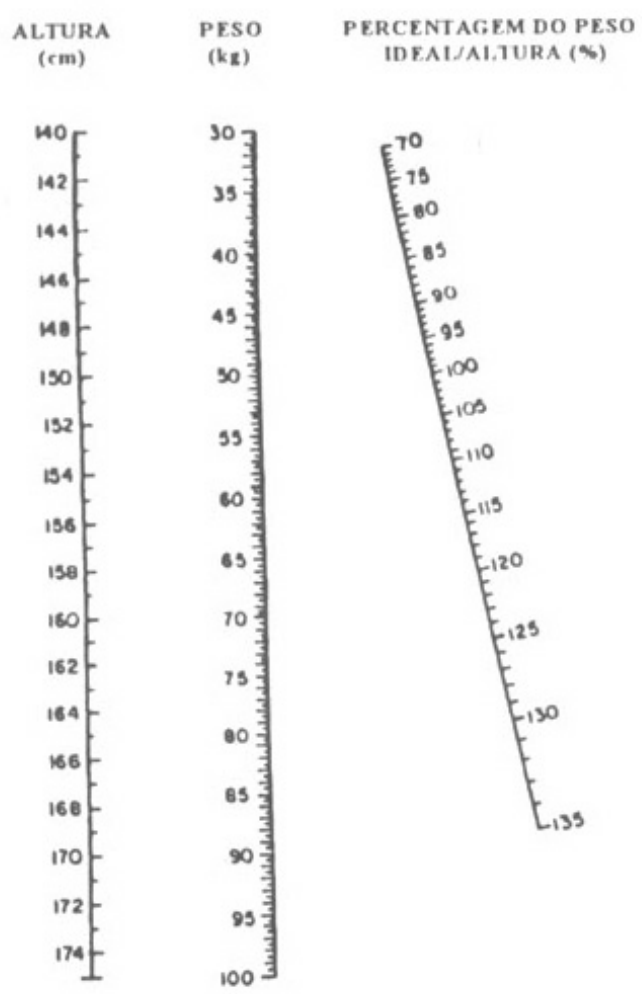

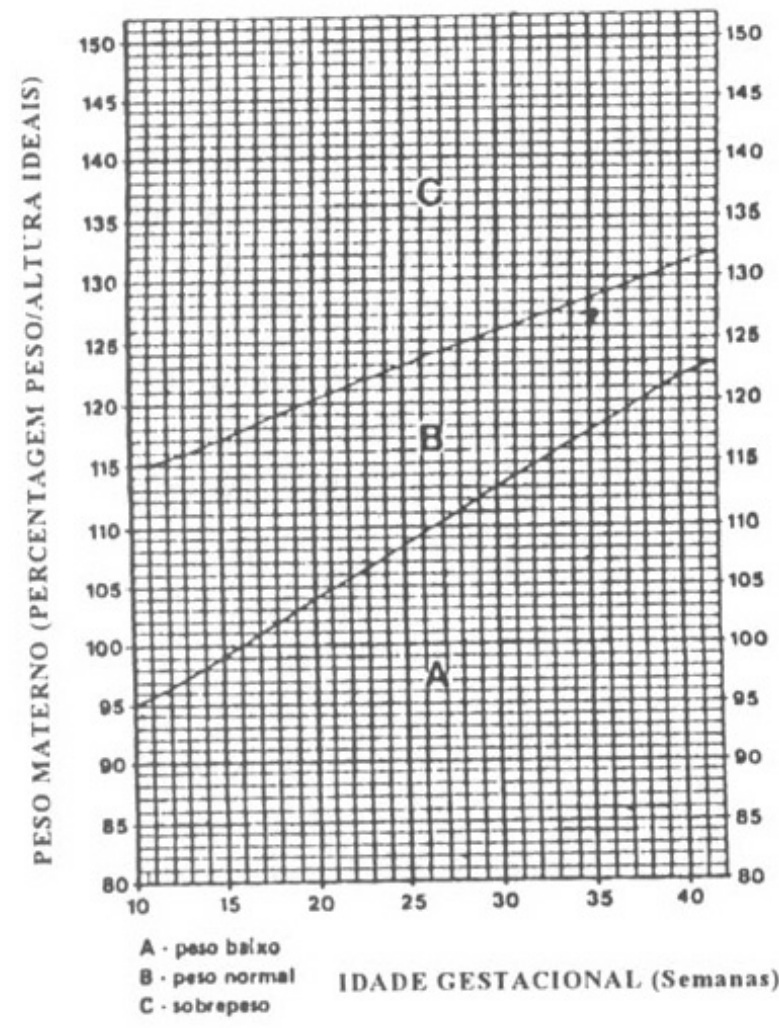

CURVA ALTURA UTERINA/IDADE GESTANCIONAL

(PARA O ACOMPANHAMENTO DO CRESCIMENTO FETAL)

ALTURA UTERINA $(\mathrm{cm})$

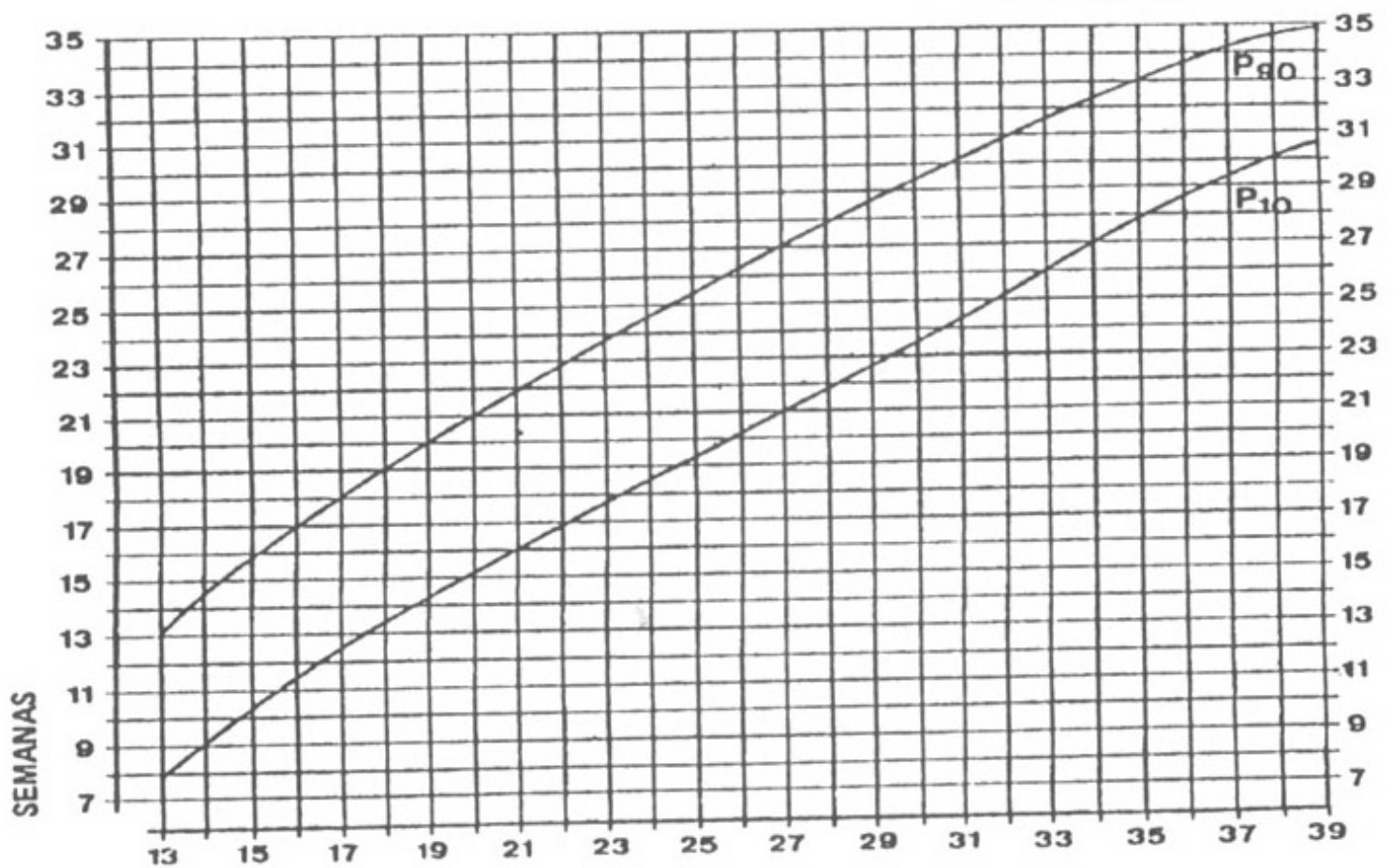


Anexo 2

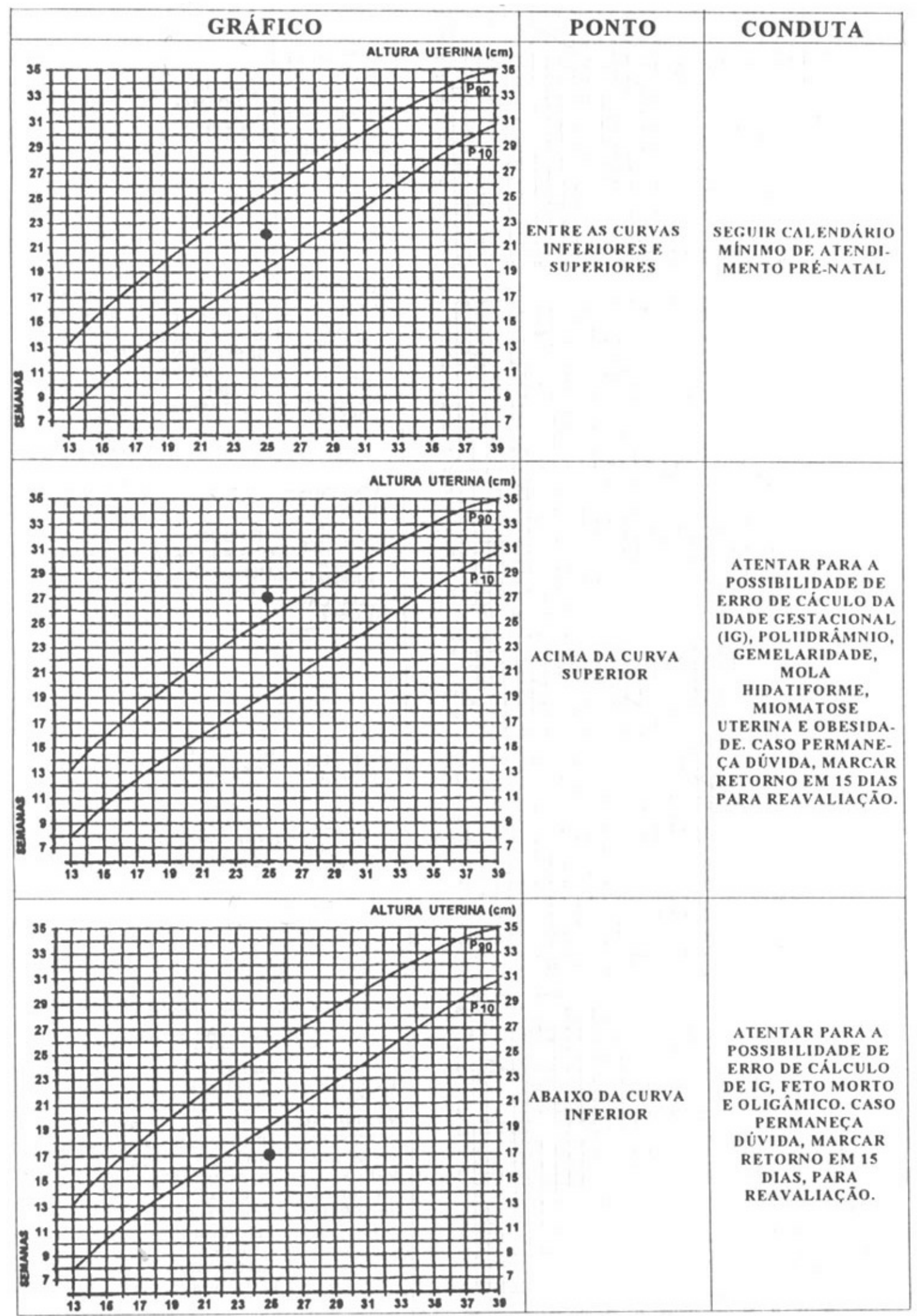




\section{Anexo 3}

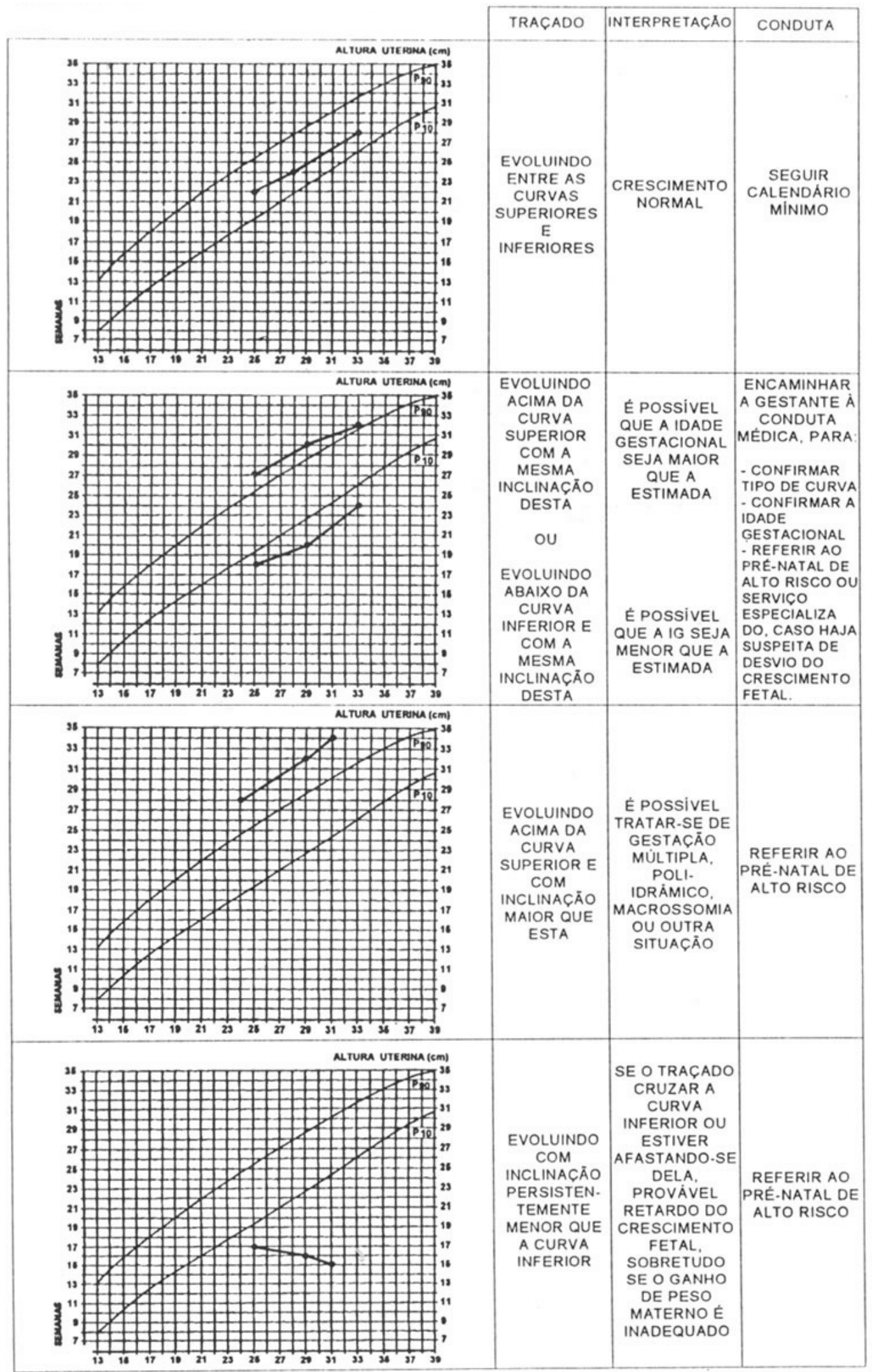

